\title{
Discovery of Dachshund 2 protein as a novel biomarker of poor prognosis in epithelial ovarian cancer
}

\author{
Björn Nodin ${ }^{1 *}$, Marie Fridberg ${ }^{1}$, Mathias Uhlén ${ }^{2,3}$ and Karin Jirström ${ }^{1}$
}

\begin{abstract}
Background: The Dachshund homolog 2 (DACH2) gene has been implicated in development of the female genital tract in mouse models and premature ovarian failure syndrome, but to date, its expression in human normal and cancerous tissue remains unexplored. Using the Human Protein Atlas as a tool for cancer biomarker discovery, $\mathrm{DACH} 2$ protein was found to be differentially expressed in epithelial ovarian cancer (EOC). Here, the expression and prognostic significance of DACH2 was further evaluated in ovarian cancer cell lines and human EOC samples.

Methods: Immunohistochemical expression of DACH2 was examined in tissue microarrays with 143 incident EOC cases from two prospective, population-based cohorts, including a subset of benign-appearing fallopian tubes $(\mathrm{n}=$ 32). A nuclear score (NS), i.e. multiplier of staining fraction and intensity, was calculated. For survival analyses, cases were dichotomized into low (NS < = 3) and high (NS > 3) using classification and regression tree analysis. Kaplan Meier analysis and Cox proportional hazards modelling were used to assess the impact of DACH2 expression on survival. DACH2 expression was analysed in the cisplatin sensitive ovarian cancer cell line A2780 and its cisplatin resistant derivative $\mathrm{A} 2780-\mathrm{Cp} 70$. The specificity of the $\mathrm{DACH} 2$ antibody was tested using siRNA-mediated silencing of DACH2 in A2780-Cp70 cells.
\end{abstract}

Results: DACH2 expression was considerably higher in the cisplatin resistant A2780-Cp70 cells compared to the cisplatin-sensitive A2780 cells. While present in all sampled fallopian tubes, DACH2 expression ranged from negative to strong in EOC. In EOC, DACH2 expression correlated with several proteins involved in DNA integrity and repair, and proliferation. DACH2 expression was significantly higher in carcinoma of the serous subtype compared to non-serous carcinoma. In the full cohort, high DACH2 expression was significantly associated with poor prognosis in univariable analysis, and in carcinoma of the serous subtype, DACH2 remained an independent factor of poor prognosis.

Conclusions: This study provides a first demonstration of DACH2 protein being expressed in human fallopian tubes and EOC, with the highest expression in serous carcinoma where DACH2 was found to be an independent biomarker of poor prognosis. Future research should expand on the role of DACH2 in ovarian carcinogenesis and chemotherapy resistance.

Keywords: $\mathrm{DACH} 2$, ovarian cancer, prognosis

\section{Background}

Epithelial ovarian cancer (EOC) is the fifth most common cause of cancer-related death in women and the leading cause of death from gynaecological malignancy [1]. Etiological factors involved in ovarian carcinogenesis remain

\footnotetext{
* Correspondence: bjorn.nodin@med.lu.se

'Department of Clinical Sciences, Division of Pathology, Lund University,

Skåne University Hospital, 22185 Lund, Sweden

Full list of author information is available at the end of the article
}

poorly defined, and effective treatment protocols are limited. The poor ratio of survival to incidence is related to the high percentage of cases diagnosed at an advanced stage, and the symptoms of EOC are often vague and overlap with other more common gastrointestinal and gynaecological diseases. Despite aggressive surgery and chemotherapy, most patients relapse within 3 to 5 years, and the median time to relapse is 15 months after diagnosis [2]. Thus, there is an urgent need for the identification

\section{() Biomed Central}


of novel diagnostic, prognostic, and predictive biomarkers for development of personalized therapeutic regimens for ovarian cancer patients.

Using the Human Protein Atlas http://www.proteinatlas.org as a tool for antibody based biomarker discovery $[3,4]$, the Dachshund 2 (DACH2) protein was identified as being differentially expressed among EOC samples, ranging from negative to strong nuclear staining. Based on this observation, we hypothesized that DACH2 might be involved in ovarian carcinogenesis and, hence, a putative prognostic and treatment predictive biomarker in EOC.

The dachshund (DACH) gene was first described in Drosophila, where it encodes a nuclear protein involved in development of the eyes, limbs and genital disc $[5,6]$. While Drosophila has a single dachshund gene, two $D A C H$ genes, $D A C H 1$ and $D A C H 2$, have been found in mice, humans and chicken [7-10] In mice, the DACH1 and $D A C H 2$ genes show functional redundancy during development of the female genital tract, whereby defects are associated with Müllerian but not Wolffian duct development [11]. In humans, the $D A C H 2$ gene has been implicated in premature ovarian failure (POF) syndrome $[12,13]$, indicating that alterations of the human DACH2 protein may constitute a risk-factor for POF by altering the correct process of ovarian follicle differentiation [13].

While the role of DACH2 in human tumourigenesis remains unexplored, alterations of DACH1 expression has been described in several cancer forms, e.g. breast [14], prostate [15], endometrial [16], gastric [17] and ovarian cancer [18]. The prognostic value of DACH1 seems to be cancer-type dependent in that reduced $\mathrm{DACH} 1$ levels have been associated with poor prognosis in breast, gastric, and endometrial cancer $[16,17,19]$ and with tumour progression in prostate cancer [15], whilst in EOC, DACH1 has been shown to be up-regulated in advance-stage ovarian cancer and promote resistance to TGF- $\beta$ signaling [18].

The aim of this study was to investigate the prognostic role of $\mathrm{DACH} 2$ protein expression in ovarian cancer, by immunohistochemical analysis of 154 EOC samples from two prospective, population-based cohorts. $\mathrm{DACH} 2$ levels were also assessed in a cisplatin sensitive and resistant ovarian cancer cell line, respectively.

\section{Methods \\ Patients}

The study cohort is a merge of all incident cases of epithelial ovarian cancers in the population-based prospective cohort studies Malmö Diet and Cancer Study ( $\mathrm{n}=101)[20]$ and Malmö Preventive Medicine Cohort $(\mathrm{n}=108)[21]$ until Dec 31st 2007. Thirty-five patients participated in both studies, and archival tumour tissue could be retrieved from 154 of the total number of 174 cases. Cases were identified from the Swedish Cancer Registry up until 31 Dec 2006, and from The Southern Swedish Regional Tumour Registry for the period of 1 Jan - 31 Dec 2007. All tumours were re-evaluated regarding histological subtype and histological grade by a board certified pathologist $(\mathrm{KJ})$. Information regarding clinical stage was obtained from the medical charts, following the standardized FIGO classification of tumour staging. Information on residual tumour after surgery was not available. Standard adjuvant therapy was platinum-based chemotherapy, from the 1990s given in combination with paclitaxel.

Histopathological, clinical and treatment data were obtained from the clinical- and/or pathology records. Information on vital status and cause of death was obtained from the Swedish Cause of Death Registry up until 31 Dec 2008. Follow-up started at date of diagnosis and ended at death, emigration or 31 Dec 2008, whichever came first. After a median follow-up of 2.65 years (range $0-21), 105$ patients $(68.2 \%)$ were dead and 49 $(31.8 \%)$ alive. Patient-and tumour characteristics of the cohort have been described in detail previously [22-24]. Ethical permissions for the MDCS (Ref. 51/90), and the present study (Ref. 530/2008), were obtained from the Ethics Committee at Lund University.

\section{Tissue microarray construction and immunohistochemistry}

Areas representative of cancer were marked on full-face haematoxylin and eosin stained sections and TMAs constructed as previously described [25]. In brief, 2-4 1.0 $\mathrm{mm}$ cores were taken from each tumour and mounted in a new recipient block using a semi-automated arraying device (TMArrayer; Pathology Devices, Inc, Westminster, MD, USA).

For immunohistochemical analysis of DACH2, $4 \mu \mathrm{m}$ TMA-sections were automatically pretreated using the PT-link system (DAKO, Glostrup, Denmark) and then stained in a Autostainer Plus (DAKO) with a polyclonal anti-DACH2 antibody (HPA0000258, Atlas Antibodies AB, Stockholm, Sweden) diluted 1:50. Immunohistochemistry for RBM3, Chek1, Chek2, MCM3, estrogen receptor $\alpha$ (ER), progesterone receptor (PR), and androgen receptor (AR) was performed as previously described [22-24]. Ki67 was analysed using a monoclonal antibody (MIB-1, DAKO, diluted 1:200)

\section{Analysis of immunohistochemical staining}

$\mathrm{DACH} 2$ was primarily expressed in the nucleus and for assessment of DACH2 expression, both the fraction of positive cells and staining intensity were taken into account. Nuclear fraction was categorized into four groups, namely 0 (0-1\%), 1 (2-25\%), 2 (26-75) and 3 $(>75 \%)$ and nuclear staining intensity denoted as $0-3$, 
whereby 0 = negative, 1 = intermediate, $2=$ moderate and 3 = strong intensity. A combined nuclear score (NS) was then constructed as a multipler of DACH2 nuclear fraction and intensity, thus ranging from 0 to 9 . Ki67 was annotated as the fraction of positive staining cells and denoted as 0 (0-1\%), 1(2-25\%), 2(26-50\%) and 3(> 50\%).

\section{Cell lines and reagents}

The human ovarian cancer cell line A2780 and the cisplatin-resistant variant A2780-Cp70 were maintained in RPMI-1640 supplemented with glutamine, 10\% fetal bovine serum and $1 \%$ pencillin/streptomycin in a humidified incubator of $5 \% \mathrm{CO} 2$ at $37^{\circ} \mathrm{C}$.

\section{Real-time quantitative PCR and Western Blotting}

Total RNA isolation (RNeasy, QIAgen, Hilden, Germany), cDNA synthesis (Reverse Transcriptase kit, Life Technologies, Carlsbad, Ca, USA) and quantitative real-time PCR (qRT-PCR) analysis of DACH2 expression with TaqMan Gene Expression Assay (Hs 00364968, Life Technologies) was performed according to the manufacturers instructions. Quantification of expression levels were calculated by using the comparative $\mathrm{Ct}$ method, normalization according to the house keeping gene 18S (s03928990 g1 RN 18S1; Life Technologies).

For immunoblotting, cells were lysed in ice-cold RIPA buffer (Cayman Chemical Company, Ann Arbor, MI, USA) and supplemented with protease inhibitor cocktail Complete Mini (Roche, Basel, Switzerland). Thirty $\mu \mathrm{g}$ of protein were separated on 4-12\% Nu-PAGE Bis-Tris gels and transferred onto iBlot Gel Transfer Stacks Nitrocellulose (Life technologies). DACH2 was detected by the polyclonal DACH2 antibody (HPA 0000258, Atlas Antibodies AB) diluted 1:250 in blocking solution, (WesternBreeze Chemiluminescent Immunodetection System (Life technologies) followed by a secondary antibody solution, Alk-Phos Conjugated, Anti-Rabbit (WesternBreeze Chemiluminescent Immunodetection System, Life technologies) and visualized using WesternBreeze Chemiluminescent Immunodetection System (Life technologies). Membranes were stripped and re-probed with an anti- $\beta$-actin antibody (Santa Cruz, Biotechnology, Santa Cruz, CA, USA) at a dilution of 1:1000, to provide a loading control.

\section{Cell pellet arrays}

Cell lines were fixed in $4 \%$ formalin and processed in gradient alcohols. Cell pellets were cleared in xylene and washed multiple times in molten paraffin. Once processed, cell lines were arrayed in duplicate $1.0 \mathrm{~mm}$ cores using a manual tissue arrayer (Beecher Inc, WI, USA) and IHC was performed on $4 \mu \mathrm{m}$ sections using the DACH2 antibody diluted 1:50.
siRNA mediated knockdown of DACH2 gene expression Transfection with siRNA against DACH2 (Life Technologies) or control siRNA (Life Technologies) was performed with Lipofectamine 2000 (Life Technologies) with a final concentration of $50 \mathrm{nM}$ siRNA. Two independent RNA oligonucleotides (s229511 and s229512, Life Technologies) targeting DACH2 were used.

\section{Statistical analysis}

Spearman's Rho test was used for comparison of DACH2 expression and clinicopathological and tumour biological characteristics. Classification regression tree (CRT) analysis was used to decide optimal cutoff for survival analysis. Kaplan-Meier analysis and log rank test were used to illustrate differences in ovarian cancer specific survival (OCSS) and overall survival (OS) according to DACH2 expression. Cox regression proportional hazards models were used for estimation of hazard ratios (HRs) for death from ovarian cancer or overall causes according to DACH2 expression in both uni- and multivariable analysis, adjusted for stage and differentiation grade. Experimental data are expressed as mean \pm SD of three independent experiments. Statistical significance of differences between means was determined by Student's t test. All calculations were performed using IBM SPSS Statistics Version 20 (SPSS Inc, Chicago, IL). All statistical tests were two-sided and a p value $<0.05$ was considered statistically significant.

\section{Results}

Antibody validation and comparison of DACH2 levels in cisplatin-sensitive vs cisplatin-resistant ovarian cancer cells

DACH2 protein expression, assessed by both IHC and Western blotting, was substantially higher in the cisplatin-resistant derivative A2780-Cp70 cells compared to the parental A2780 cells, in which the DACH2 protein was barely detectable (Figure $1 \mathrm{~A}$ and $1 \mathrm{~B}$ ). Real-time quantitative PCR (qRT-PCR) confirmed a similar difference whereby there was a 3.7-fold higher level of DACH2 mRNA in the A2780-Cp70 compared to the A2780 cell line (Figure $1 \mathrm{C}$ ). Cisplatin resistance in the A2780-Cp70 cells relative to the A2780 cells has been confirmed previously [23]. The specificity of the DACH2 antibody was confirmed by siRNA-mediated knockdown of DACH2 in A2780/Cp70 cells. IHC performed on formalin fixed, paraffin embedded siRNA transfected A2780/Cp70 cells revealed a marked decrease in immunoreactivity in the DACH2 knockdown cells compared to controls as visualized by IHC on cell pellets (Figure 1D).

\section{Immunohistochemical expression of DACH2 in fallopian tubes and EOC}

Following antibody optimisation and staining, DACH2 expression could be evaluated in $32 / 38$ (84.2\%) samples 


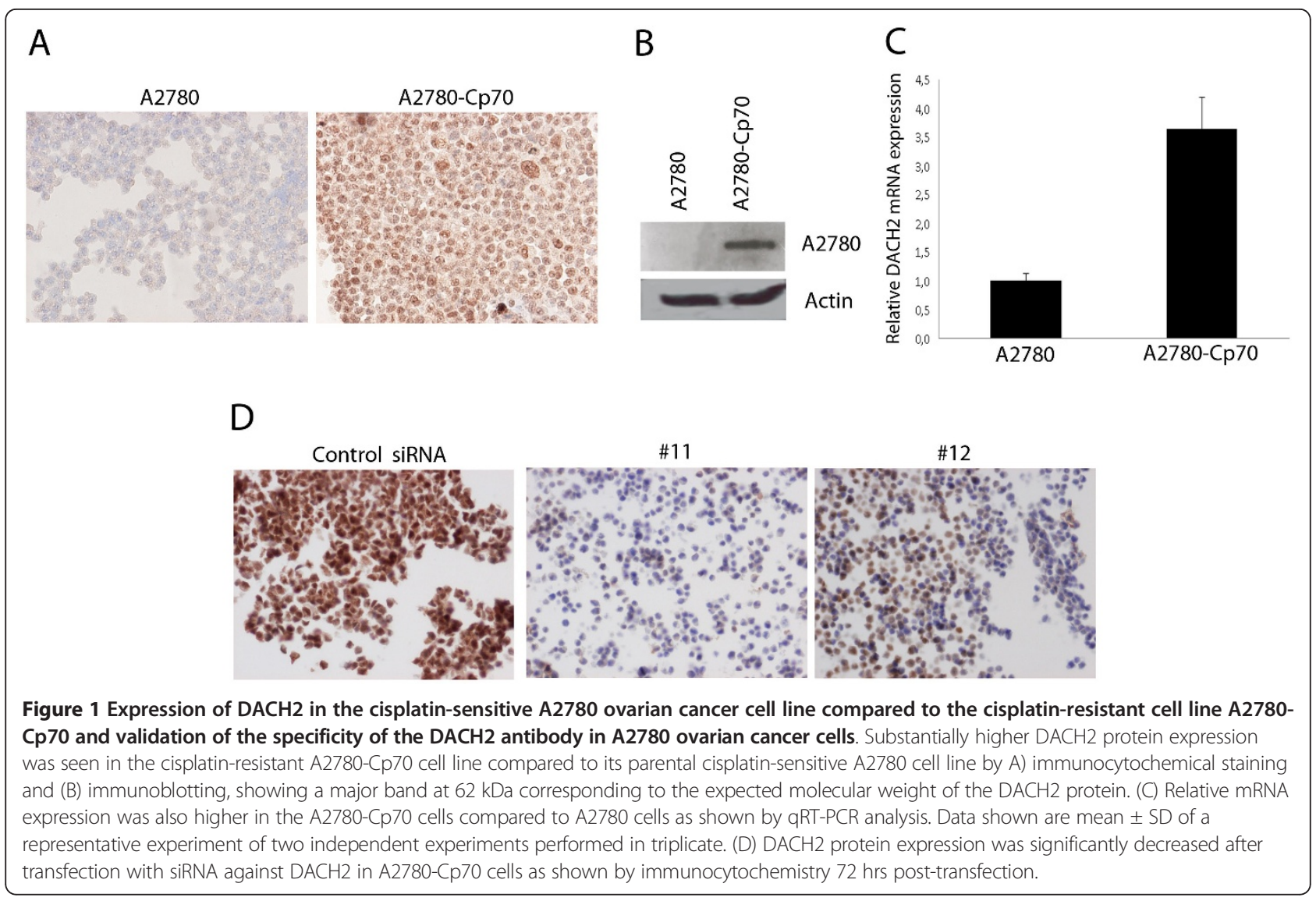

from fallopian tubes and 143/154 (92.9\%) EOC cases. There was no obvious heterogeneity in $\mathrm{DACH} 2$ expression between duplicate TMA cores. Images representing different patterns of expression in tubal epithelium and EOC are shown in Figure $2 \mathrm{~A}-\mathrm{H}$, whereby A-B represent tubal epithelium, C-E tumours with a NS $<=3$ and F-H tumours with a NS $>3$. As regards the staining distribution, expression of $\mathrm{DACH} 2$ protein was evident in all fallopian tubes with nuclear scores ranging from 3-9 (Figure 3A). A wider range of DACH2 expression was observed in EOC, where 8 (5.6\%) cases were denoted as $\mathrm{DACH} 2$ negative and $33(23.1 \%)$ cases had a NS < 3, e.g. lower than in the tubal epithelium (Figure 3B). There was however no statistically significant difference in $\mathrm{DACH} 2$ expression in tubal epithelium and EOC in cases from which paired samples had been analysed $(\mathrm{n}=30)$, of whom 2 had DACH2 negative tumours, 5 had tumours with a NS $<3$, and the remaining cases $(n=25)$ had a NS $>=3$ in the invasive component (data not shown). There was no significant difference in DACH2 expression between cancer located to the ovaries and metastatic deposits (data not shown). DACH2 staining was significantly higher in carcinomas of the serous subtype compared to nonserous carcinomas $(\mathrm{R}=0.244, \mathrm{p}=0.003$ ) (Figure $3 \mathrm{C}$ ). Comparison of different histological subtypes within non-serous carcinomas, i.e. mucinous, endometroid and clear cell carcinomas revealed no significant difference in the distribution of DACH2 staining (data not shown). The distribution of DACH2 in tubal epithelium was similar in serous and non-serous carcinomas (data not shown).

\section{Association between DACH2 expression, clinicopathological characteristics and markers of proliferation and DNA integrity}

Next, we examined the relationship between DACH2 expression (NS) and established clinicopathological and investigative parameters (Table 1). In the full cohort, $\mathrm{DACH} 2$ expression showed a positive correlation to Ki67, Chk1, Chk2 and MCM3 expression. There was no significant correlation between DACH2 expression and established clinicopathological factors, i.e. clinical stage and grade, nor to RBM3, AR, ER or PR expression. In the serous subtype, DACH2 was not significantly associated with any other clinicopathological or tumour biological parameters (Table 1).

\section{Association between $\mathrm{DACH} 2$ expression and survival from EOC}

CRT analysis suggested an optimal cutoff point at NS > 3 to determine the impact of $\mathrm{DACH} 2$ expression on 

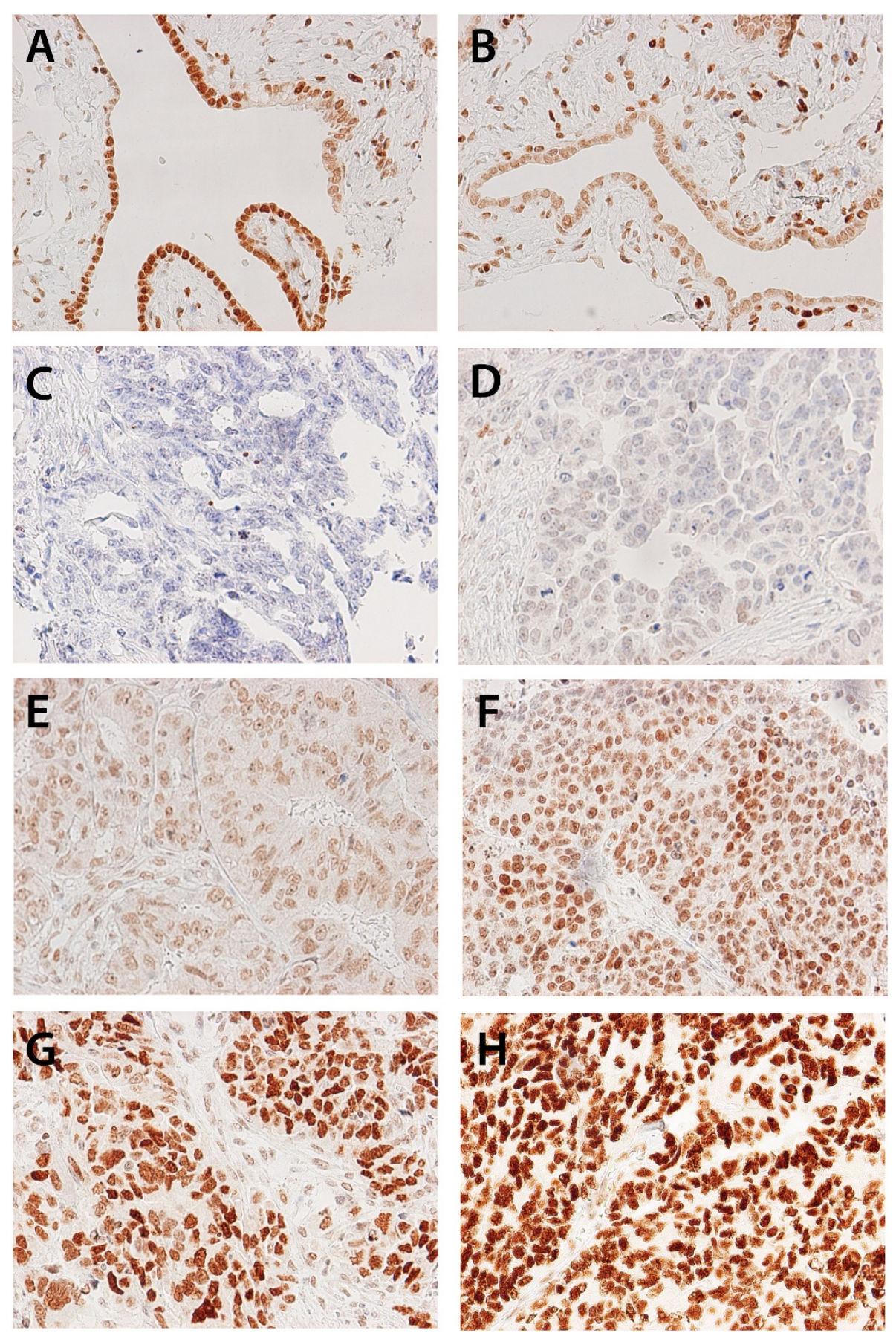

Figure 2 Immunohistochemical images of DACH2 staining in fallopian tubes and ovarian cancer. Images (20x magnification) representing immunohistochemical expression of DACH2 in (A, B) fallopian tubes, and EOC ranging from (C) negative, (D) weak intensity in few cells, (E) weak intensity in majority of cells, $(F)$ moderate to strong intensity in majority of cells, $(G)$ strong intensity in majority of cells and $(H)$ strong intensity in all tumour cells.

OCSS and OS. Kaplan Meier analysis of the entire cohort $(\mathrm{n}=143)$ demonstrated a significantly reduced OCSS $(p=0.046)$ and OS $(p=0.021)$ for tumours expressing high levels of DACH2 (Figure 4A, B). These associations were accentuated in the subgroup of serous carcinoma $(\mathrm{n}=84)$ for both OCSS $(\mathrm{p}=0.008)$ and OS $(\mathrm{p}=0.004)$ (Figure $4 \mathrm{C}, \mathrm{D})$. The associations between $\mathrm{DACH} 2$ expression and survival were confirmed in univariable Cox regression analysis (Table 2). In multivariable analysis, $\mathrm{DACH} 2$ remained an independent 


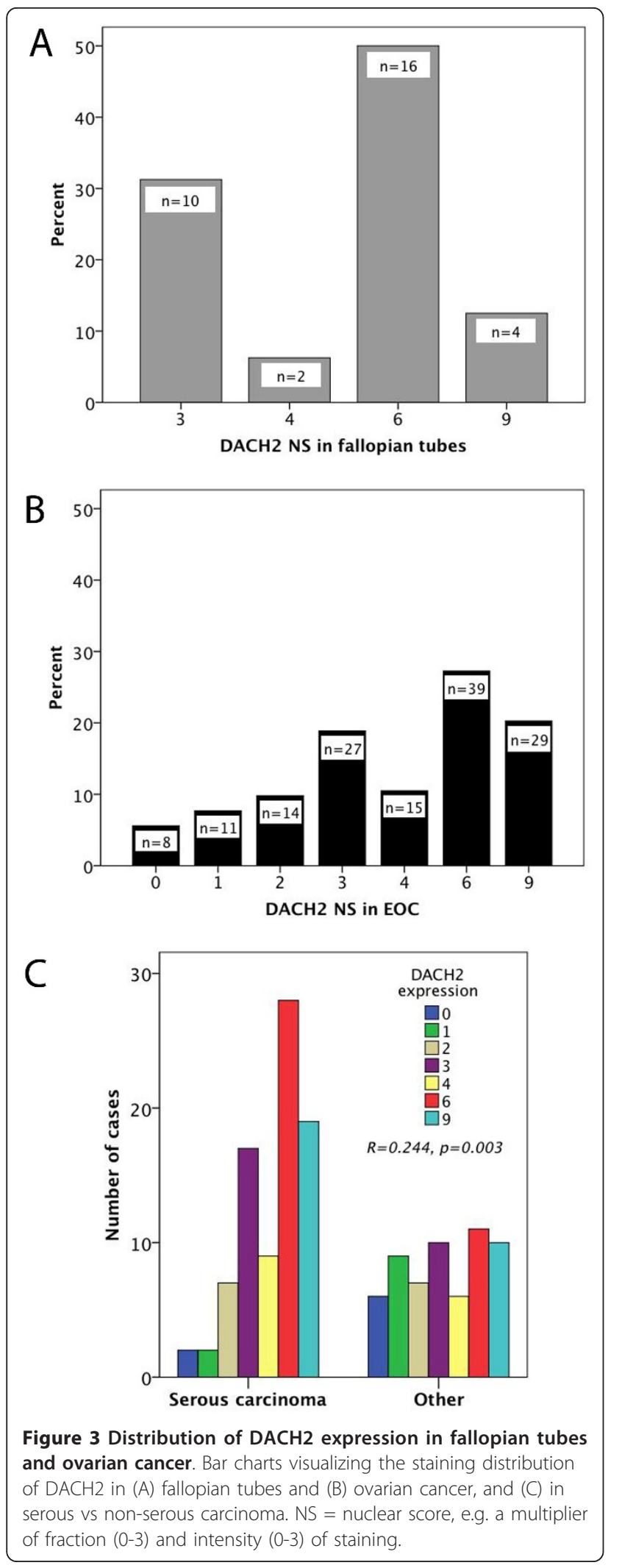

Table 1 Associations between DACH2 expression and clinicopathological parameters in all patients and patients with serous carcinoma.

\begin{tabular}{|c|c|c|}
\hline Factor & $\begin{array}{c}\text { All } \\
\text { DACH2 }\end{array}$ & $\begin{array}{c}\text { Serous carcinoma } \\
\text { DACH2 }\end{array}$ \\
\hline \multicolumn{3}{|l|}{ Age } \\
\hline R & 0.044 & 0.078 \\
\hline$p$ & 0.603 & 0.483 \\
\hline $\mathrm{n}$ & 143 & 84 \\
\hline \multicolumn{3}{|c|}{ Differentiation grade } \\
\hline $\mathrm{R}$ & $-0,031$ & -0.189 \\
\hline$p$ & 0.716 & 0.086 \\
\hline$n$ & 143 & 84 \\
\hline \multicolumn{3}{|c|}{ Clinical stage } \\
\hline $\mathrm{R}$ & 0.025 & 0.078 \\
\hline$p$ & 0.779 & 0.494 \\
\hline$n$ & 131 & 80 \\
\hline \multicolumn{3}{|l|}{ Ki67 } \\
\hline $\mathrm{R}$ & 0.208 & 0.086 \\
\hline$p$ & $0.013^{*}$ & 0.435 \\
\hline $\mathrm{n}$ & 141 & 84 \\
\hline \multicolumn{3}{|l|}{$\overline{A R}$} \\
\hline $\mathrm{R}$ & 0.028 & -0.045 \\
\hline$p$ & 0.738 & 0.683 \\
\hline$n$ & 143 & 84 \\
\hline \multicolumn{3}{|l|}{ ER } \\
\hline $\mathrm{R}$ & 0.122 & -0.067 \\
\hline$p$ & 0.151 & 0.554 \\
\hline$n$ & 139 & 81 \\
\hline \multicolumn{3}{|l|}{$\overline{P R}$} \\
\hline $\mathrm{R}$ & 0.130 & 0.085 \\
\hline$p$ & 0.126 & 0.437 \\
\hline$n$ & 141 & 85 \\
\hline \multicolumn{3}{|l|}{ RBM3 } \\
\hline $\mathrm{R}$ & $-0,072$ & -0.167 \\
\hline$p$ & 0.393 & 0.129 \\
\hline $\mathrm{n}$ & 141 & 84 \\
\hline \multicolumn{3}{|l|}{ Chek1 } \\
\hline $\mathrm{R}$ & 0.194 & 0.139 \\
\hline$p$ & $0.024^{*}$ & 0.225 \\
\hline$n$ & 134 & 78 \\
\hline \multicolumn{3}{|l|}{ Chek2 } \\
\hline $\mathrm{R}$ & 0.182 & 0.155 \\
\hline$p$ & $0.032^{*}$ & 0.164 \\
\hline $\mathrm{n}$ & 139 & 78 \\
\hline \multicolumn{3}{|l|}{ MCM3 } \\
\hline $\mathrm{R}$ & 0.252 & 0.104 \\
\hline$p$ & $0.003^{* *}$ & 0.360 \\
\hline $\mathrm{n}$ & 134 & 79 \\
\hline
\end{tabular}

$\mathrm{R}=$ Spearman's correlation coefficient, $\mathrm{p}=\mathrm{p}$-value, $\mathrm{n}=$ number of cases available for analysis. $E R=$ estrogen receptor, $\mathrm{PR}=$ progesterone receptor, $\mathrm{AR}$ $=$ Androgen receptor. ${ }^{*}$ significance at $5 \%$ level, ** significance at $1 \%$ level. The analysis are based on multipliers of staining intensity and fraction (nuclear score) for DACH2, RBM3, Chek1, Chek2 and MCM3 and categories of nuclear fraction for Ki67, AR, ER, and PR. 


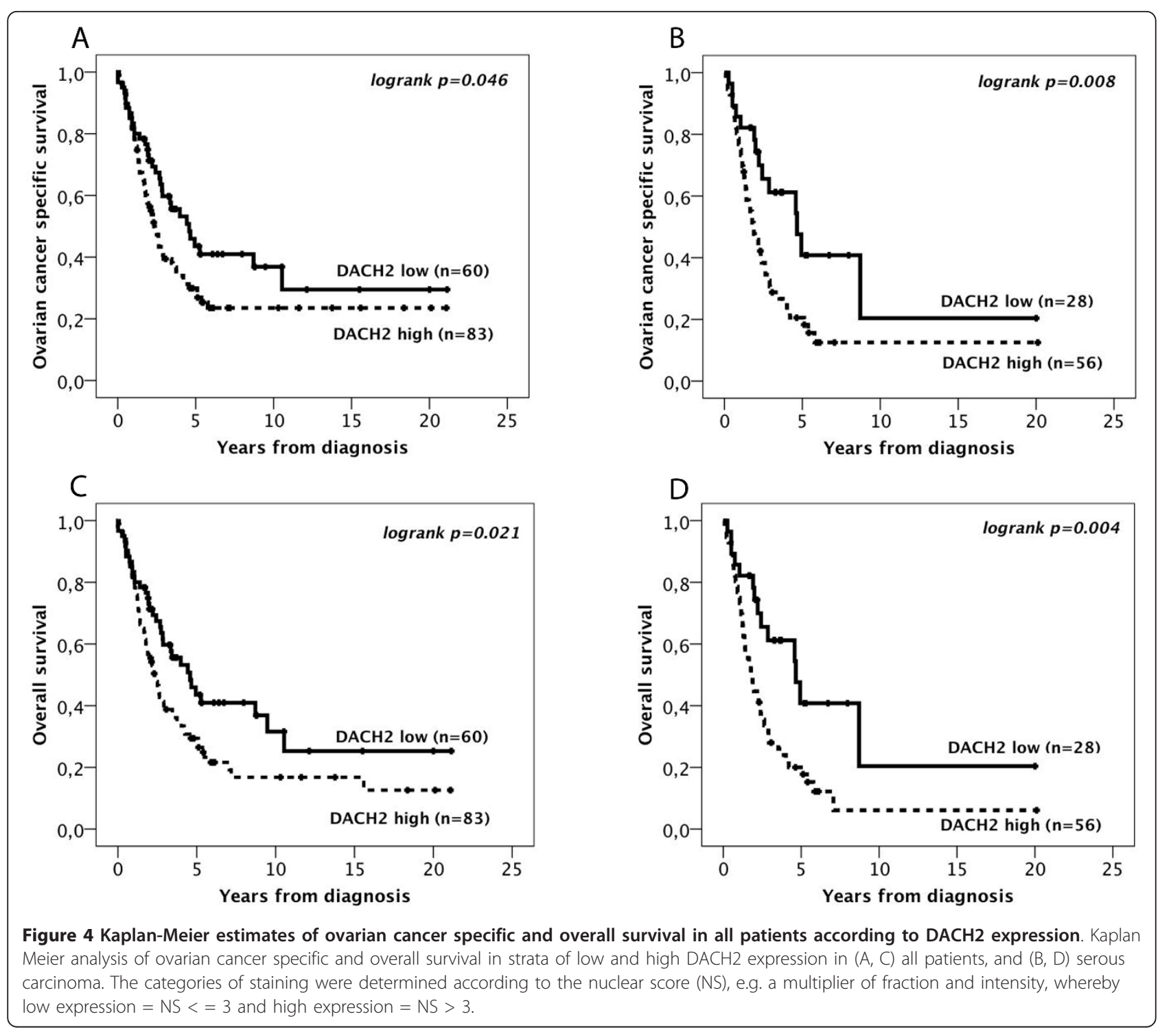

prognostic factor in patients with serous carcinoma for both OCSS $(\mathrm{HR}=2.01,95 \%$ CI 1.05-3.85, $\mathrm{p}=0.035)$ and OS $(\mathrm{HR}=2.13,95 \%$ CI 1.12-4.08, $\mathrm{p}=0.022)$, but not in the full cohort (Table 2). DACH2 was not prognostic in separate analysis of other histological subgroups (data not shown). Ki67 expression was not prognostic, neither in the full cohort nor in the subgroup of serous carcinoma (data not shown).

\section{Discussion}

The results from this study provide a first demonstration of DACH2 being abundantly expressed at the protein level in human fallopian tubes and EOC. Moreover, $\mathrm{DACH} 2$ expression was found to be significantly higher in EOC of the serous subtype compared to non-serous carcinoma, and an independent predictor of poor survival in the former.
In the full cohort of EOC, there was a positive correlation between expression of $\mathrm{DACH} 2$ and crucial checkpoint proteins and regulators of cellular DNA damage response Chek1 and Chek2 [26], as well as MCM3, a key component of the DNA replication licensing system [27]. High expression of Chek1, Chek2 and MCM3 has previously been demonstrated to be associated with a poor prognosis in the here studied cohort of tumours, although not independent of other established clinicopathological parameters [24]. Moreover, the positive association between DACH2 and Ki67 further supports a role for $\mathrm{DACH} 2$ in conferring a more malignant phenotype in EOC.

The association of DACH2 expression with proteins involved in maintenance of DNA integrity might suggest a role for $\mathrm{DACH} 2$ in chemotherapy resistance, a notion further supported by the finding of substantially higher 
Table 2 Relative risks of death from ovarian cancer and overall death according to DACH2 expression in all patients and patients with serous carcinoma.

\begin{tabular}{|c|c|c|c|c|c|c|}
\hline & \multicolumn{3}{|c|}{ Ovarian cancer specific survival } & \multicolumn{3}{|c|}{ Overall survival } \\
\hline & $\mathrm{HR}(95 \% \mathrm{Cl})$ & $p$-value & $n$ (events) & $\mathrm{HR}(95 \% \mathrm{Cl})$ & p-value & n(events) \\
\hline All & & Univariable & & & Univariable & \\
\hline DACH2 low & 1,00 & 0.048 & 60 & 1,00 & 0.022 & 60 \\
\hline \multirow[t]{2}{*}{$\mathrm{DACH} 2$ high } & $1.54(1.00-2.35)$ & & 83 & $1.63(1.07-2.47)$ & & 83 \\
\hline & & Multivariable & & & Multivariable & \\
\hline DACH2 low & 1,00 & 0.182 & 55 & 1,00 & 0.088 & 55 \\
\hline DACH2 high & 1.36(0.87-2.12) & & 76 & 1.57(0.95-2.28) & & 76 \\
\hline Serous carcinoma & & Univariable & & & Univariable & \\
\hline $\mathrm{DACH} 2$ low & 1,00 & 0.010 & 28 & 1,00 & 0.005 & 28 \\
\hline \multirow[t]{2}{*}{$\mathrm{DACH} 2$ high } & $2.21(1.21-4.04)$ & & 56 & $2.34(1.23-4.26)$ & & 56 \\
\hline & & Multivariable & & & Multivariable & \\
\hline DACH2 low & 1,00 & 0.035 & 25 & 1,00 & 0.022 & 25 \\
\hline $\mathrm{DACH} 2$ high & $2.01(1.05-3.85)$ & & 51 & $2.13(1.12-4.08)$ & & 51 \\
\hline
\end{tabular}

Cox uni- and multivariable analysis of relative risks of death from ovarian cancer and overall death according to DACH2 expression in all patients and patients with serous carcinoma. HR = Hazard ratio. The categories of staining were determined according to the nuclear score (NS), e.g. a multiplier of fraction and intensity, whereby low expression $=$ NS $<=3$ and high expression $=$ NS $>3$. Multivariate analysis included adjustment for differentiation grade (low-intermediate vs high) and clinical stage (1-2 vs 3 and 4).

$\mathrm{DACH} 2$ expression levels in the cisplatin resistant A2780-Cp70 compared to cisplatin sensitive A2780 ovarian cancer cells. It would therefore be of interest to address the molecular basis for how DACH2 might modulate the effects of both platinum and taxane-based chemotherapy in future mechanistic studies. However, the association between DACH2 expression and other investigative markers, e.g. Chek1, Chek2, MCM3 and Ki67, was only evident in the full cohort and not in the subgroup of serous carcinoma, where $\mathrm{DACH} 2$ expression was significantly higher than in non-serous carcinomas, and an independent factor of poor prognosis. These findings, together with the various important developmental functions demonstrated for DACH proteins, not least related to the female genital tract [11-13], indicate that DACH2 might play a more important role in EOC development than in chemotherapy resistance. As DACH2 was found to be expressed in the epithelium of all concomitantly sampled benign-appearing fallopian tubes and a significant proportion of serous carcinomas have been suggested to arise within the fimbrial tubal epithelium [28-30], these observations could indicate differential roles of DACH2 in the progression of serous and nonserous carcinomas, respectively.

While DACH1 has been demonstrated to co-localize with ER in breast cancer and AR in normal prostate and exert repressive effects on both ER and AR mediated signaling [14,15], no correlation was found between expression of $\mathrm{DACH} 2$ and $\mathrm{AR}, \mathrm{ER}$ or $\mathrm{PR}$ in the here examined EOC cohort. However, these findings do not exclude a role for $\mathrm{DACH} 2$ as a mediator of endocrine signaling in EOC.
Apart from providing a first description of the expression and prognostic significance of $\mathrm{DACH} 2$ in EOC, this is also, to our knowledge, the first report of DACH2 expression in any human cancer form. This illustrates the utility of the Human Protein Atlas as a tool for antibody-based biomarker discovery [3], not least in light of the lack of well-validated antibodies in translational research, but also since it facilitates the selection of hypotheses relevant to human disease. The specificity of the polyclonal antibody generated against $\mathrm{DACH} 2$ within the HPA project was here further validated by a marked reduction of $\mathrm{DACH} 2$ expression in formalinfixed, paraffin-embedded siDACH2 treated EOC cells compared to controls, confirming its suitability for use in immunohistochemical biomarker studies. Although being a semi-quantitative method, immunohistochemistry has several advantages compared to other assays, not least in the clinical setting, as it is simple to perform, fast and comparatively cheap. More importantly, it allows for marker analysis in different subcellular locations, which might be of crucial importance for prognostication and treatment stratification of patients.

Interestingly, loss of DACH1 expression has been associated with poor prognosis in all hitherto investigated cancer forms with the exception of ovarian cancer, where gene expression profiling analysis identified $\mathrm{DACH} 1$ to be up-regulated in advance-stage ovarian cancer and to inhibit TGF- $\beta$ signaling in ovarian cancer cells [18]. Whether DACH2 is prognostic in other cancer forms, and to what extent this might be cancer-type specific, will be of interest to determine in future studies. 


\section{Conclusions}

Using an antibody-based biomarker discovery approach, DACH2 has been identified as a novel biomarker of poor prognosis in EOC. Future studies are warranted to confirm these findings in additional patient cohorts and to further elucidate the role of DACH2 in ovarian carcinogenesis, progression and chemotherapy response.

\section{List of abbreviations}

DACH2: Dachshund2; EOC: Epithelial ovarian cancer; NS:Nuclear score; OCSS: Ovarian cancer specific survival; OS: Overall survival; AR: Androgen receptor; ER: Estrogen receptor $a$; PR: Progesterone receptor.

\section{Acknowledgements}

We thank Prof Robert Brown, Imperial College, London, for kindly providing the A2780 and A2780- Cp70 cells. This study was supported by grants from the Knut and Alice Wallenberg Foundation, the Swedish Cancer Society, Gunnar Nilsson's Cancer Foundation, Region Skåne and the Research Funds of Skåne University Hospital.

\section{Author details}

'Department of Clinical Sciences, Division of Pathology, Lund University, Skåne University Hospital, 22185 Lund, Sweden. ${ }^{2}$ Department of Proteomics, AlbaNova University Center, Royal Institute of Technology, 10691 Stockholm, Sweden. ${ }^{3}$ Science for Life Laboratory, Royal Institute of Technology, 10691 Stockholm, Sweden.

\section{Authors' contributions}

BN performed statistical analysis, carried out the experimental studies and drafted the manuscript. MF assisted with the experimental studies and helped to draft the manuscript, MU participated in the design of the study and technical assistance. KJ conceived of the study and participated in its design and coordination and helped to draft the manuscript. All authors read and approved the final manuscript.

\section{Competing interests}

A patent application has been filed related to the use of $\mathrm{DACH} 2$ as a prognostic and treatment predictive biomarker in EOC.

Received: 22 December 2011 Accepted: 27 January 2012 Published: 27 January 2012

\section{References}

1. Jemal A, Siegel R, Ward E, Hao Y, Xu J, Thun MJ: Cancer statistics, 2009. CA Cancer J Clin 2009, 59(4):225-249.

2. Hennessy BT, Coleman RL, Markman M: Ovarian cancer. Lancet 2009, 374(9698):1371-1382.

3. Ponten $F$, Jirstrom $K$, Uhlen M: The Human Protein Atlas-a tool for pathology. J Pathol 2008, 216(4):387-393.

4. Bjorling E, Lindskog C, Oksvold P, Linne J, Kampf C, Hober S, Uhlen M, Ponten F: A web-based tool for in silico biomarker discovery based on tissue-specific protein profiles in normal and cancer tissues. Mol Cell Proteomics 2008, 7(5):825-844.

5. Keisman EL, Baker BS: The Drosophila sex determination hierarchy modulates wingless and decapentaplegic signaling to deploy dachshund sex-specifically in the genital imaginal disc. Development 2001, 128(9):1643-1656.

6. Mardon G, Solomon NM, Rubin GM: Dachshund encodes a nuclear protein required for normal eye and leg development in Drosophila. Development 1994, 120(12):3473-3486.

7. Davis RJ, Shen W, Heanue TA, Mardon G: Mouse Dach, a homologue of Drosophila dachshund, is expressed in the developing retina, brain and limbs. Development genes and evolution 1999, 209(9):526-536.

8. Kozmik Z, Pfeffer P, Kralova J, Paces J, Paces V, Kalousova A, Cvekl A: Molecular cloning and expression of the human and mouse homologues of the Drosophila dachshund gene. Development genes and evolution 1999, 209(9):537-545.
9. Heanue TA, Davis RJ, Rowitch DH, Kispert A, McMahon AP, Mardon G, Tabin CJ: Dach1, a vertebrate homologue of Drosophila dachshund, is expressed in the developing eye and ear of both chick and mouse and is regulated independently of Pax and Eya genes. Mechanisms of development 2002, 111(1-2):75-87.

10. Davis RJ, Shen W, Sandler YI, Heanue TA, Mardon G: Characterization of mouse Dach2, a homologue of Drosophila dachshund. Mechanisms of development 2001, 102(1-2):169-179.

11. Davis RJ, Harding M, Moayedi Y, Mardon G: Mouse Dach1 and Dach2 are redundantly required for Mullerian duct development. Genesis 2008, 46(4):205-213.

12. Prueitt RL, Chen $H$, Barnes Rl, Zinn AR: Most X;autosome translocations associated with premature ovarian failure do not interrupt X-linked genes. Cytogenet Genome Res 2002, 97(1-2):32-38.

13. Bione S, Rizzolio F, Sala C, Ricotti R, Goegan M, Manzini MC, Battaglia R, Marozzi A, Vegetti W, Dalpra L, et al: Mutation analysis of two candidate genes for premature ovarian failure, DACH2 and POF1B. Hum Reprod 2004, 19(12):2759-2766.

14. Popov VM, Zhou J, Shirley LA, Quong J, Yeow WS, Wright JA, Wu K, Rui H, Vadlamudi RK, Jiang J, et al: The cell fate determination factor DACH1 is expressed in estrogen receptor-alpha-positive breast cancer and represses estrogen receptor-alpha signaling. Cancer Res 2009, 69(14):5752-5760.

15. Wu K, Katiyar S, Witkiewicz A, Li A, McCue P, Song LN, Tian L, Jin M, Pestell RG: The cell fate determination factor dachshund inhibits androgen receptor signaling and prostate cancer cellular growth. Cancer Res 2009, 69(8):3347-3355.

16. Nan F, Lu Q, Zhou J, Cheng L, Popov VM, Wei S, Kong B, Pestell RG, Lisanti MP, Jiang J, et al: Altered expression of DACH1 and cyclin D1 in endometrial cancer. Cancer Biol Ther 2009, 8(16):1534-1539.

17. Yamada Y, Arao T, Gotoda T, Taniguchi H, Oda I, Shirao K, Shimada Y, Hamaguchi T, Kato K, Hamano T, et al: Identification of prognostic biomarkers in gastric cancer using endoscopic biopsy samples. Cancer Sci 2008, 99(11):2193-2199.

18. Sunde JS, Donninger H, Wu K, Johnson ME, Pestell RG, Rose GS, Mok SC, Brady J, Bonome T, Birrer MJ: Expression profiling identifies altered expression of genes that contribute to the inhibition of transforming growth factor-beta signaling in ovarian cancer. Cancer Res 2006 66(17):8404-8412.

19. Wu K, Li A, Rao M, Liu M, Dailey V, Yang Y, Di Vizio D, Wang C, Lisanti MP Sauter $G$, et al: DACH1 is a cell fate determination factor that inhibits cyclin D1 and breast tumour growth. Mol Cell Biol 2006, 26(19):7116-7129.

20. Berglund G, Elmstahl S, Janzon L, Larsson SA: The Malmo Diet and Cancer Study. Design and feasibility. J Intern Med 1993, 233(1):45-51.

21. Berglund G, Eriksson KF, Israelsson B, Kjellstrom T, Lindgarde F, Mattiasson I, Nilsson JA, Stavenow L: Cardiovascular risk groups and mortality in an urban swedish male population: the Malmo Preventive Project. J Intern Med 1996, 239(6):489-497.

22. Nodin B, Zendehrokh N, Brandstedt J, Nilsson E, Manjer J, Brennan DJ, Jirstrom K: Increased androgen receptor expression in serous carcinoma of the ovary is associated with an improved survival. $J$ Ovarian Res 3:14.

23. Ehlen A, Brennan DJ, Nodin B, O'Connor DP, Eberhard J, AlvaradoKristensson $M$, Jeffrey IB, Manjer J, Brandstedt J, Uhlen $M$, et al: Expression of the RNA-binding protein RBM3 is associated with a favourable prognosis and cisplatin sensitivity in epithelial ovarian cancer. J Trans/ Med 8:78.

24. Ehlen A, Nodin B, Rexhepaj E, Brandstedt J, Uhlen M, AlvaradoKristensson M, Ponten F, Brennan DJ, Jirstrom K: RBM3-regulated genes promote DNA integrity and affect clinical outcome in epithelial ovarian cancer. Translational oncology 2011, 4(4):212-221.

25. Kononen J, Bubendorf L, Kallioniemi A, Barlund M, Schraml P, Leighton S, Torhorst J, Mihatsch MJ, Sauter G, Kallioniemi OP: Tissue microarrays for high-throughput molecular profiling of tumour specimens. Nat Med 1998, 4(7):844-847.

26. Kastan MB, Bartek J: Cell-cycle checkpoints and cancer. Nature 2004 432(7015):316-323

27. Bailis JM, Forsburg SL: MCM proteins: DNA damage, mutagenesis and repair. Current opinion in genetics \& development 2004, 14(1):17-21.

28. Dubeau L: The cell of origin of ovarian epithelial tumours. Lancet Oncol 2008, 9(12):1191-1197. 
29. Carlson JW, Miron A, Jarboe EA, Parast MM, Hirsch MS, Lee Y, Muto MG, Kindelberger D, Crum CP: Serous tubal intraepithelial carcinoma: its potential role in primary peritoneal serous carcinoma and serous cancer prevention. J Clin Oncol 2008, 26(25):4160-4165.

30. Lee Y, Miron A, Drapkin R, Nucci MR, Medeiros F, Saleemuddin A, Garber J, Birch C, Mou H, Gordon RW, et al: A candidate precursor to serous carcinoma that originates in the distal fallopian tube. J Pathol 2007, 211(1):26-35.

doi:10.1186/1757-2215-5-6

Cite this article as: Nodin et al:: Discovery of Dachshund 2 protein as a novel biomarker of poor prognosis in epithelial ovarian cancer. Journal of Ovarian Research 2012 5:6.

Submit your next manuscript to BioMed Central and take full advantage of:

- Convenient online submission

- Thorough peer review

- No space constraints or color figure charges

- Immediate publication on acceptance

- Inclusion in PubMed, CAS, Scopus and Google Scholar

- Research which is freely available for redistribution

Submit your manuscript at www.biomedcentral.com/submit 